\title{
Congestive hepatopathy after Fontan operation and related factors assessed by transient elastography
}

\author{
Byung Won Yoo, MD, ${ }^{\mathrm{a}}$ Jae Young Choi, MD, PhD, ${ }^{\mathrm{b}}$ Lucy Youngmin Eun, MD, PhD, \\ Han Ki Park, MD, PhD, ${ }^{c}$ Young Hwan Park, MD, ${ }^{c}$ and Seung Up Kim, MD ${ }^{d, e, f}$
}

\begin{abstract}
Objective: Congestive hepatopathy after a Fontan operation can have a major effect on long-term morbidity. We evaluated congestive hepatopathy in patients with Fontan circulation using transient elastography to determine which risk factors for hepatopathy are related to liver stiffness (LS).
\end{abstract}

\begin{abstract}
Methods: We evaluated 46 patients with Fontan circulation and 26 with right side heart failure (RHF) and hepatic congestion using laboratory tests, the aspartate aminotransferase-to-platelet ratio index, ultrasonography, and transient elastography.

Results: The LS value was significantly greater in the Fontan group $(21.1 \pm 8.0 \mathrm{kPa})$ than in the RHF group $(10.0 \pm 9.0 \mathrm{kPa})$. The total bilirubin and albumin serum levels, white blood cell count, and aspartate aminotransferase to platelet ratio index correlated significantly with LS in the Fontan group. Of the risk factors, age at evaluation $(\mathrm{r}=0.42, P=.004)$, age at Fontan completion $(\mathrm{r}=0.51, P<.001)$, inferior vena cava diameter $(\mathrm{r}=0.35$, $P=.02)$, and spleen size $(\mathrm{r}=0.53, P=.002)$ correlated significantly with LS. Nineteen patients in the Fontan group $(41.3 \%)$ had abnormal ultrasound findings, and the frequency of abnormal findings increased with increasing LS $(P=.002)$. In the subgroup with the greatest LS value $(\geq 30 \mathrm{kPa}), 88.9 \%$ had abnormal ultrasound findings and $44.4 \%$ liver cirrhosis. Multivariate analysis revealed that age at Fontan procedure completion and total bilirubin were independent risk factors for hepatopathy.
\end{abstract}

Conclusions: The present study revealed that congestive hepatopathy developed in a significant fraction of patients with long-term Fontan circulation and that transient elastography could be an easy and useful method to assess congestive hepatopathy in these patients. (J Thorac Cardiovasc Surg 2014;148:1498-505)

First introduced in 1968, the Fontan operation and its modifications have been established as definitive palliation in patients with single ventricular physiology. However, nonpulsatile passive flow to the pulmonary vascular bed resulting from the absence of a pulmonary pump can cause various complications in the long term. ${ }^{1-4}$ With the improvement in surgical outcomes from the Fontan operation, recent studies have focused on late morbidities, especially hepatic complications in long-term survivors.

From the Department of Clinical Pharmacology, ${ }^{\text {a }}$ Clinical Trial Center, Severance Hospital, Yonsei University College of Medicine, Seoul, Republic of Korea; Division of Pediatric Cardiology, ${ }^{\mathrm{b}}$ Yonsei University College of Medicine, Seoul, Republic of Korea; Division of Cardiovascular Surgery, ${ }^{\mathrm{c}}$ Congenital Heart Disease Center, Severance Cardiovascular Hospital, and Department of Thoracic and Cardiovascular Surgery, Yonsei University College of Medicine, Seoul, Republic of Korea; Department of Internal Medicine, ${ }^{\mathrm{d}}$ Yonsei University College of Medicine, Seoul, Republic of Korea; Institute of Gastroenterology, ${ }^{\text {e }}$ Yonsei University College of Medicine, Seoul, Republic of Korea; and Liver Cirrhosis Clinical Research Center, ${ }^{\mathrm{f}}$ Seoul, Republic of Korea.

Disclosures: Authors have nothing to disclose with regard to commercial support.

Drs H.K.P. and S.U.K. contributed equally to the present study.

Received for publication Dec 16, 2013; revisions received March 28, 2014; accepted for publication April 4, 2014; available ahead of print May 10, 2014

Address for reprints: Seung Up Kim, MD, Department of Internal Medicine, Yonsei

University College of Medicine, Yonsei University Health System, 50 Yonsei-Ro,

Seodaemun-gu, Seoul 120-752, Republic of Korea (E-mail: ksukorea@yuhs.ac).

$0022-5223 / \$ 36.00$

Copyright (c) 2014 by The American Association for Thoracic Surgery

http://dx.doi.org/10.1016/j.jtcvs.2014.04.010
Regarding hepatopathy after the Fontan operation, it has been suggested that chronic passive congestion of the liver due to elevated systemic venous pressure produces histologic changes and hepatic dysfunction, predominantly causing coagulopathy and cholestasis. ${ }^{5-7}$ It has been reported that congestive hepatopathy in these patients could progress to irreversible liver injury, liver cirrhosis, and hepatocellular carcinoma, ${ }^{6-8}$ which would have a major effect on longterm morbidity and mortality. However, the prevalence and risk factors of liver fibrosis after the Fontan operation have been largely unknown. In addition, it can be difficult for physicians to judge the extent of congestive hepatopathy after the Fontan operation, because it behaves in a distinct fashion from inflammatory liver disease. The clinical symptoms are not obvious, and the laboratory tests, imaging studies, and several serologic indexes for liver fibrosis can show minor and nonspecific changes, even with marked fibrosis. The absence of a sensitive and reliable modality to assess and monitor the hepatic changes in Fontan patients has made early diagnosis difficult.

Although liver biopsy has been the reference standard for the assessment of liver fibrosis, ${ }^{9}$ limitations, such as discomfort, rare but fatal complications, sampling variability, ${ }^{10,11}$ and intra- and interobserver interpretational variability, have significantly limited the wide use of liver biopsy, even when significant fibrosis is suspected. Recently, transient elastography (TE; FibroScan, 


\author{
Abbreviations and Acronyms \\ $\mathrm{APRI}=$ aspartate aminotransferase to platelet ratio \\ index \\ IVC = inferior vena cava \\ LS = liver stiffness \\ RHF $=$ right side heart failure \\ $\mathrm{TE}=$ transient elastography
}

Echosens, Paris, France) has been developed for noninvasive assessment of liver stiffness (LS), a surrogate marker of liver fibrosis. TE has been actively validated in patients with various chronic liver diseases and has been proved to be a sensitive and accurate tool for the determination of the severity of liver fibrosis. ${ }^{12,13}$ The volume of liver parenchyma that can be studied by TE is approximately 100 times greater than that obtained by biopsy, resulting in a potentially lower sampling error. ${ }^{14}$ According to studies of patients with chronic viral hepatitis, TE will be useful for predicting the risk of hepatocellular carcinoma development and monitoring the treatment response, as well as in directly assessing liver fibrosis. ${ }^{15}$ From this evidence, TE has recently been recommended by the European Association for the Study of the Liver Clinical Practice Guidelines for the first-line assessment of liver fibrosis in patients with hepatitis C. ${ }^{16}$ However, few studies have investigated whether TE can be used to evaluate congestive hepatopathy, especially in patients with Fontan circulation. ${ }^{17}$

In the present study, we evaluated congestive hepatopathy in patients with Fontan circulation using TE and investigated whether TE can assess congestive hepatopathy in these patients. We also aimed to determine the risk factors related to the presence of LS.

\section{METHODS \\ Subjects}

From March 2011 to February 2012, we evaluated congestive hepatopathy using TE and other noninvasive methods in 49 patients with $>5$ years follow-up after Fontan operation (Fontan group). We also evaluated congestive hepatopathy in 26 patients who had hepatic congestion caused by right side heart failure (RHF) to compare the severity of the hepatopathy between the 2 groups. The patients in the RHF group were defined as subjects who had significant right ventricular volume overload and/or systolic dysfunction on their cardiac magnetic resonance imaging scan, resulting in hepatic congestion for $>5$ years. The hepatic congestion can be identified by echocardiography when it shows inferior vena cava (IVC) dilatation (at the site just proximal to the junction of the hepatic veins, $>21 \mathrm{~mm}$ in diameter) and collapse of the IVC when the patient breathed in or sniffed (collapsibility $<50 \%$ ). ${ }^{18}$ We excluded patients from both groups with known hepatobiliary disease (including viral hepatitis and alcoholic liver disease) and acute decompensated heart failure.

The institutional review board of Severance Hospital approved the present study, and the patients provided verbal informed consent.

\section{Transient Elastography}

TE, a simple noninvasive method, measures the propagation velocity of the elastic shear wave induced by vibration using the Doppler technique. Because the velocity of the shear wave increases with the stiffness of the liver parenchyma, TE can estimate the LS, a surrogate marker of liver fibrosis. ${ }^{12}$

TE was performed on the right lobe of the liver through the intercostal spaces on patients lying in the dorsal decubitus position with the right arm in maximal abduction. ${ }^{15,19}$ The operator located a liver portion that was $\geq 6$ $\mathrm{cm}$ thick and free of large vascular structures and pressed the probe button to begin the measurement. All TE measurements were performed by 1 experienced technician who had performed $>10,000$ examinations and was unaware of the clinical data of the patients. The results are expressed in kilopascals. In the present study, only LS values with $\geq 10$ validated measurements and a success rate of $\geq 60 \%$ were considered reliable. The median value of the successful measurements was selected as representative of the LS value in a given patient only if the interquartile range to median value ratio was $<0.3$. Any LS value that did not satisfy these conditions was considered unreliable and was excluded from additional analysis.

\section{Laboratory Tests and Other Noninvasive Assessments}

Laboratory tests, including hematologic tests (hemoglobin, white blood cell count, platelet count), biochemical tests (alanine aminotransferase, aspartate aminotransferase, total bilirubin, total protein, serum albumin), and coagulation tests (prothrombin time, expressed as the international normalized ratio, partial activated thromboplastin time), were performed in both groups. Using the laboratory test results, the serum marker of hepatic fibrosis, the aspartate aminotransferase to platelet ratio index (APRI) score [(aspartate aminotransferase/upper limit of normal) $\times 100 /$ platelet count $\left.\left(10^{9} / \mathrm{L}\right)\right]$, was calculated.

Abdominal ultrasonography was performed in the Fontan group within 3 months before or after the LS measurement. Liver cirrhosis was diagnosed clinically from the ultrasound findings and the following criteria: (1) platelet count $<100,000 / \mu \mathrm{L}$ and ultrasound findings suggestive of cirrhosis, including a blunted, nodular liver edge accompanied by splenomegaly (>12 cm); (2) esophageal or gastric varices; and/or (3) overt complications of liver cirrhosis, including ascites, variceal bleeding, and hepatic encephalopathy. ${ }^{15}$ If gastric endoscopy is not available, severe splenomegaly can be regarded as evidence of portal hypertension despite esophageal or gastric varices.

Using echocardiography, the systemic ventricular EF was determined using the $\mathrm{M}$ mode and fractional area curve. The IVC diameter was measured at the site just proximal to the junction of the hepatic veins as the maximum diameter.

\section{Statistical Analysis}

The demographic and clinical data were compared between the 2 groups using the Student $t$ test for continuous data and the chi-square test for nonnumeric parameters. Correlations between the LS values and other variables and between the LS values and the frequency of abnormal ultrasound findings were assessed using Pearson's correlation for numeric parameters and the chi-square test for non-numeric parameters. To identify the independent factors associated with the LS value, linear regression analysis was used. All clinical and laboratory parameters with statistical significance in a univariate model were included in the multivariate model analysis. The Statistical Package for Social Sciences for Windows, version 18.0 (SPSS, Inc, Chicago, Ill) was used for data analysis. Two-sided probability values were used.

\section{RESULTS}

\section{Subject and TE Findings}

Clinical data from the Fontan and RHF groups are listed in Tables 1 and 2. The assessment using FibroScan failed in 
TABLE 1. Patient characteristics of Fontan group $(n=46)$

\begin{tabular}{|c|c|}
\hline Characteristic & Value \\
\hline \multicolumn{2}{|l|}{ Age at completion of Fontan procedure (y) } \\
\hline Mean \pm standard deviation & $5.04 \pm 5.74$ \\
\hline Range & $1.2-23.1$ \\
\hline \multicolumn{2}{|l|}{ Duration of Fontan circulation (y) } \\
\hline Mean \pm standard deviation & $13.5 \pm 5.0$ \\
\hline Range & $6.5-29.0$ \\
\hline \multicolumn{2}{|l|}{ Major diagnosis } \\
\hline Pulmonary atresia & $17(37.0)$ \\
\hline Double outlet of right ventricle & $13(28.2)$ \\
\hline Tricuspid atresia & $8(17.4)$ \\
\hline Functional single ventricle & $5(10.9)$ \\
\hline Miscellaneous & $3(6.5)$ \\
\hline Isomerism (combined) & $12(26.1)$ \\
\hline \multicolumn{2}{|l|}{ Systemic ventricle } \\
\hline Right ventricle & $19(41.3)$ \\
\hline Left ventricle & $24(52.2)$ \\
\hline Uncertain & $3(6.5)$ \\
\hline \multicolumn{2}{|l|}{ Type of Fontan operation } \\
\hline Atriopulmonary connection & $7(15.2)$ \\
\hline Lateral tunnel & $14(30.4)$ \\
\hline Extracardiac conduit & $25(54.4)$ \\
\hline Systemic to pulmonary shunt (before Fontan) & $27(58.7)$ \\
\hline Fenestration & $27(58.7)$ \\
\hline \multicolumn{2}{|l|}{ Oxygen saturation $(\%)$} \\
\hline Mean \pm standard deviation & $94.8 \pm 1.9$ \\
\hline Range & $90.0-99.0$ \\
\hline Arrhythmia & $5(10.9)$ \\
\hline Heart failure medication & $30(65.2)$ \\
\hline \multicolumn{2}{|l|}{ NYHA functional class } \\
\hline I & $40(87.0)$ \\
\hline II & $6(13.0)$ \\
\hline
\end{tabular}

1 Fontan patient because of narrow intercostal spaces. We also excluded 2 Fontan patients with thick subcutaneous fat in the abdominal wall because their interquartile range to median value ratio was $>0.3$. In the remaining 46 patients in the Fontan group and 26 patients in the RHF group, the LS assessment was successful within 5 minutes with good reliability (interquartile range/ measurement $<0.3)$.

\section{Comparison Between Fontan and RHF Groups}

The clinical and laboratory variables between the Fontan and RHF groups were compared (Table 3). The serum level of total bilirubin, international normalized ratio, and partial activated thromboplastin time were significantly elevated in the Fontan group compared with the RHF group (Table 3). However, even in the Fontan group, the absolute values of these parameters were only slightly elevated or were within the normal range.

The LS values were significantly greater in the Fontan group $(21.1 \pm 8.0 \mathrm{kPa}$; median, 19.0) than in the RHF group
TABLE 2. Patient characteristics of RHF group $(n=26)$

\begin{tabular}{|c|c|c|c|}
\hline Major diagnosis & RHF etiology & $\begin{array}{c}\text { RVEDVi } \\
\geq 140 \mathrm{~mL} / \mathrm{m}^{2}(\mathrm{n})\end{array}$ & $\begin{array}{c}\text { RVEF } \\
<45 \%(\mathbf{n})\end{array}$ \\
\hline \multicolumn{4}{|l|}{$\begin{array}{l}\text { Repaired TOF } \\
\qquad(\mathrm{n}=19)\end{array}$} \\
\hline & Severe PR $(n=14)$ & 13 & 3 \\
\hline & Severe PR + TR $(n=3)$ & 2 & 1 \\
\hline & Severe TR $(n=2)$ & 2 & \\
\hline \multicolumn{4}{|l|}{ VSD/PS $(n=2)$} \\
\hline & Severe PR $(n=1)$ & 1 & \\
\hline & Severe PR + TR $(n=1)$ & 1 & \\
\hline \multicolumn{4}{|l|}{$\operatorname{ASD}(n=2)$} \\
\hline & Severe TR $(n=2)$ & 2 & \\
\hline \multicolumn{4}{|l|}{$\begin{array}{l}\text { DORV with Rastelli } \\
\text { operation }(\mathrm{n}=1)\end{array}$} \\
\hline & Severe PR $(n=1)$ & & 1 \\
\hline \multicolumn{4}{|l|}{$\operatorname{VSD}(n=1)$} \\
\hline & Severe TR $(n=1)$ & 1 & \\
\hline \multicolumn{4}{|l|}{ TGA $(\mathrm{n}=1)$} \\
\hline & Severe TR + MR $(n=1)$ & 1 & \\
\hline Arrhythmia & $3(11.5)$ & & \\
\hline HF medication & $11(42.3)$ & & \\
\hline
\end{tabular}

Data in parentheses are percentages, unless otherwise noted. $R H F$, Right heart failure; $R V E D V i$, right ventricular end-diastolic volume index; $R V E F$, right ventricular ejection fraction; TOF, tetralogy of Fallot; $P R$, pulmonary regurgitation; $T R$, tricuspid regurgitation; VSD, ventricular septal defect; $P S$, pulmonary stenosis; $A S D$, atrial septal defect; $D O R V$, double outlet of right ventricle; $T G A$, transposition of great arteries; $M R$, mitral regurgitation; $H F$, heart failure.

(10.0 $\pm 9.0 \mathrm{kPa}$; median, 6.9; $P<.001$; Figure 1). In contrast, a difference in the ARPI score was identified between the 2 groups. Although 1 patient in the RHF group who had had longstanding severe tricuspid regurgitation and complete atrioventricular conduction block had the greatest LS value among all the patients $(48.8 \mathrm{kPa})$, most patients in the RHF group had a mild to moderate LS elevation. In contrast, most of the patients in the Fontan group had a severe elevation in $\operatorname{LS}(>10 \mathrm{kPa})$.

\section{Correlations Between LS and Other Variables in the Fontan Group}

Of the demographic and clinical factors, age at evaluation (r $=0.42, P=.004$ ), age at the completion of the Fontan procedure $(\mathrm{r}=0.51, P<.001)$, and IVC diameter (r $=0.35, P=.021$ ) showed a significant correlation with the LS value (Figure 2). However, the type of Fontan procedure and the morphology of the systemic ventricle did not show any relationship with the LS value in the chi-square test. In addition, the duration of the Fontan circulation and the ejection fraction of the systemic ventricle did not show any relationship with the LS value.

Of the laboratory variables, the total bilirubin level and APRI score showed a significant correlation with the LS value, although they were not remarkably elevated beyond the normal range (total bilirubin, $\mathrm{r}=0.36, P=.013$; APRI, $\mathrm{r}=0.39, P=.009$; Figure 2). Also, the white blood cell count and the serum albumin level showed a significant 
TABLE 3. Comparison between Fontan and RHF groups

\begin{tabular}{|c|c|c|c|}
\hline Variable & $\begin{array}{c}\text { Fontan group } \\
(n=46)\end{array}$ & $\begin{array}{l}\text { RHF group } \\
\quad(n=26)\end{array}$ & $\begin{array}{c}P \\
\text { value } \\
\end{array}$ \\
\hline Gender & & & .78 \\
\hline Male & 24 & 13 & \\
\hline Female & 22 & 13 & \\
\hline Age at evaluation (y) & $18.2 \pm 7.6$ & $24.3 \pm 11.0$ & .005 \\
\hline $\operatorname{BSA}\left(\mathrm{m}^{2}\right)$ & $1.51 \pm 0.27$ & $1.62 \pm 0.18$ & .57 \\
\hline BMI $\left(\mathrm{kg} / \mathrm{m}^{2}\right)$ & $20.1 \pm 3.7$ & $21.2 \pm 3.0$ & .19 \\
\hline \multicolumn{4}{|l|}{ Echocardiographic findings } \\
\hline IVC diameter (mm) & $20.6 \pm 5.0$ & $24.9 \pm 5.9$ & .002 \\
\hline $\begin{array}{c}\text { EF of systemic } \\
\text { ventricle }(\%)\end{array}$ & $58.2 \pm 7.9$ & $63.3 \pm 7.6$ & .008 \\
\hline \multicolumn{4}{|l|}{ Laboratory test results } \\
\hline WBC count $\left(\times 10^{3} / \mu \mathrm{L}\right)$ & $6.48 \pm 1.81$ & $6.91 \pm 2.50$ & .47 \\
\hline Platelet count $\left(\times 10^{3} / \mu \mathrm{L}\right)$ & $217.1 \pm 96.4$ & $219.8 \pm 57.4$ & .92 \\
\hline AST (IU/L) & $25.3 \pm 7.0$ & $26.4 \pm 11.6$ & .67 \\
\hline ALT (IU/L) & $21.0 \pm 9.1$ & $21.7 \pm 11.2$ & .81 \\
\hline Total bilirubin (mg/dL) & $1.13 \pm 0.59$ & $0.67 \pm 0.32$ & .01 \\
\hline Albumin (g/dL) & $4.51 \pm 0.43$ & $4.23 \pm 0.53$ & .06 \\
\hline INR & $1.13 \pm 0.09$ & $1.04 \pm 0.14$ & .006 \\
\hline aPTT (s) & $34.4 \pm 4.3$ & $31.5 \pm 3.9$ & .03 \\
\hline APRI & $0.40 \pm 0.19$ & $0.37 \pm 0.20$ & .64 \\
\hline LS measurement $(\mathrm{kPa})^{*}$ & $21.1 \pm 8.0(19.0)$ & $10.0 \pm 9.0(6.9)$ & $<.001$ \\
\hline IQR/M (\%) & $17.25 \pm 9.83$ & $18.17 \pm 7.82$ & .78 \\
\hline
\end{tabular}

Data presented as mean \pm standard deviation (median) or $\mathrm{n}$. $R H F$, Right side heart failure; $B S A$, body surface area; $B M I$, body mass index; $I V C$, inferior vena cava; $E F$, ejection fraction; $W B C$, white blood cell; $A S T$, aspartate aminotransferase; $A L T$, alanine aminotransferase; $I N R$, international normalized ratio; $a P T T$, partial activated thromboplastin time; $A P R I$, AST to platelet ratio index; $L S$, liver stiffness; $I Q R / M$, interquartile range to median value of LS ratio. *Normal range recognized as $\leq 5.5 \mathrm{kPa}^{23}$

inverse correlation with the LS value (white blood cell count, $\mathrm{r}=-0.41, P=.006$; albumin, $\mathrm{r}=-0.31, P=.038)$.

The results of the multivariate analysis showed that patient age at the completion of the Fontan procedure $(\beta=0.58, P=.009)$ and the total bilirubin levels ( $\beta=0.58, P=.003$ ) were independent factors associated with the LS value.

\section{Ultrasound Findings and LS in the Fontan Group}

Of the 46 patients in the Fontan group, $19(41.3 \%)$ had abnormal ultrasound findings, including liver cirrhosis in $4(8.7 \%)$, increased coarse echogenicity of the liver parenchyma, representing diffuse liver disease in 14 (30.4\%), and a hepatic nodule with increased hepatic echogenicity in $1(2.2 \%)$. The frequency of abnormal ultrasound findings increased with an increasing $\mathrm{LS}$ value $(P=.002$; Figure 3$)$. Of 9 patients with high LS values of $\geq 30 \mathrm{kPa}, 8(88.9 \%)$ had abnormal ultrasound findings. Also, all the patients with liver cirrhosis $(n=4,44.4 \%)$ were included in this subgroup with a high LS value of $\geq 30 \mathrm{kPa}$.

The splenic size was measured in 30 patients in the Fontan group, excluding 12 patients with asplenia or polysplenia syndrome and 4 who had not had a clear window for the

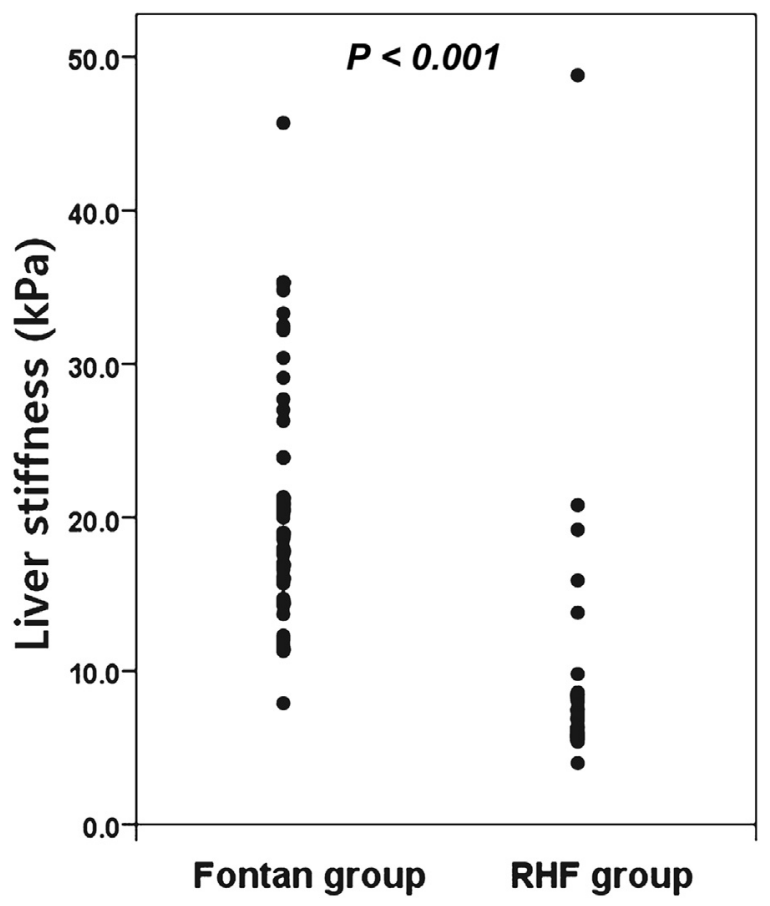

FIGURE 1. Distribution of liver stiffness values for Fontan and right side heart failure $(R H F)$ groups.

spleen. The spleen size in the 30 patients showed a close correlation with the LS value ( $\mathrm{r}=0.53, P=.002$, Figure 2$)$.

In the RHF group, 4 patients, whose LS value was $>15$ $\mathrm{kPa}$, had undergone ultrasonography. In those patients (including 1 patient with an LS value of $48 \mathrm{kPa}$ ), no abnormal finding, except for mild hypoechogenicity of the liver, was observed.

\section{DISCUSSION}

Although few studies have used TE in patients with Fontan circulation, our results have revealed that TE can be used to evaluate congestive hepatopathy after the Fontan operation. The LS values correlated well with the results of other noninvasive assessments in the patients with Fontan circulation. The total bilirubin level, APRI score (the APRI score was increased according to the LS value without the effect of cyanosis and reactive thrombocytosis), spleen size, and frequency of abnormal ultrasound findings correlated significantly with the LS values. This might indicate that TE can sensitively identify the hepatic status even in patients with well-preserved liver function similar to that of our study population. Although the APRI score, a simple well-known fibrosis prediction model and a first line screening tool for hepatic fibrosis in patients with hepatitis C, ${ }^{20,21}$ had shown a significant correlation with hepatopathy after the Fontan operation in a previous study, ${ }^{22}$ it was nearly normal and statistically equivalent in both groups in our study. However, in contrast, the LS values in all 

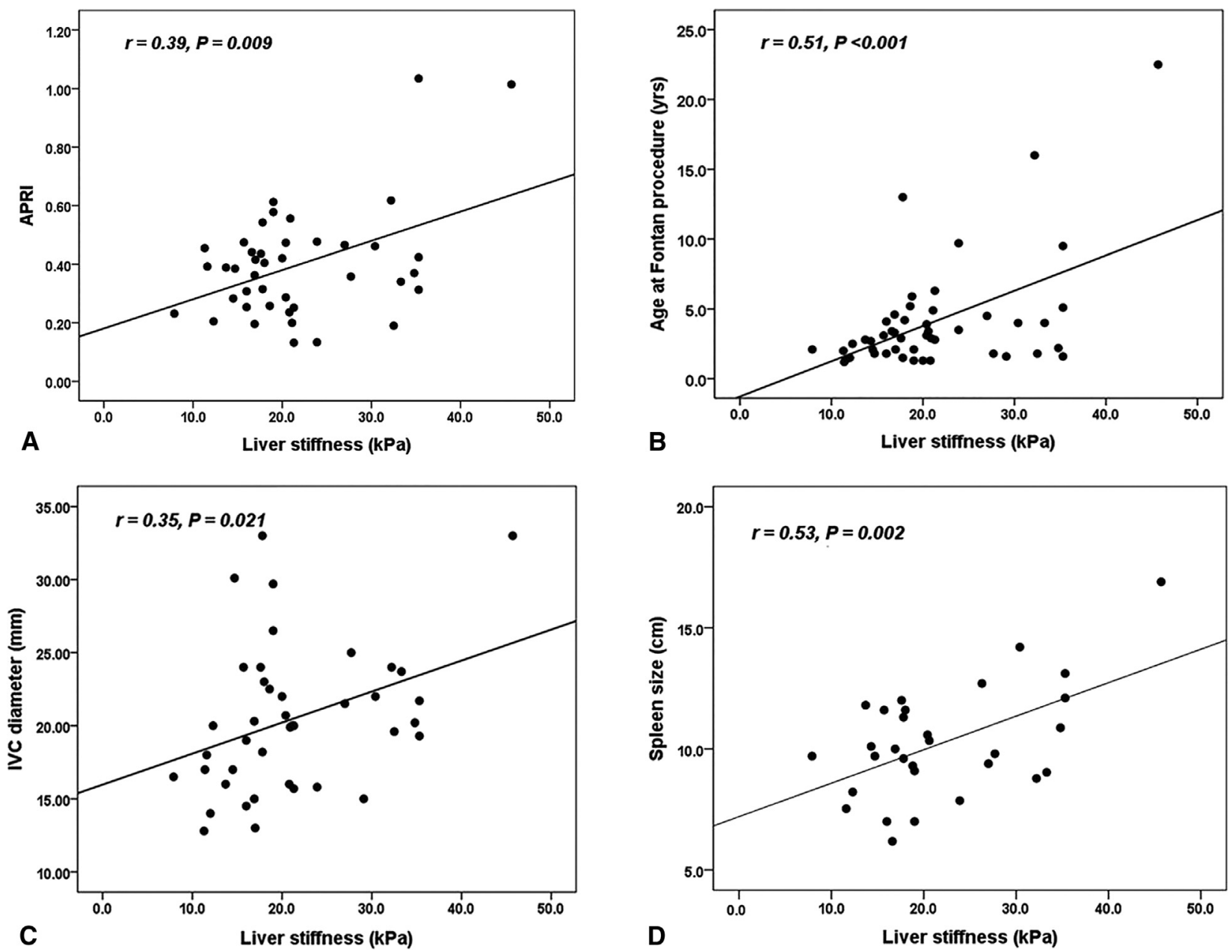

FIGURE 2. Correlations between the liver stiffness values and related clinical factors. APRI, Aspartate aminotransferase to platelet ratio index; IVC, inferior vena cava.

patients with Fontan circulation were strikingly greater than the recognized normal range $(\leq 5.5 \mathrm{kPa})^{23}$ and those of patients with RHF. Also, remembering that even patients diagnosed with liver cirrhosis using abdominal ultrasonography showed minor abnormalities in the laboratory test results and APRI score, but high LS values, the difference in the TE results and other assessments might indicate the differentiability of TE in the silent progression of congestive hepatopathy after the Fontan operation.

TE definitely has a limitation in differentiating liver fibrosis from hepatic congestion; thus, hepatic congestion has been considered a significant confounder in assessing primary liver disease using TE. ${ }^{24,25}$ However, when we evaluate patients with Fontan circulation, the finding of hepatic congestion is not information that should be discarded. Because the LS values paradoxically reflect hepatic congestion, they can provide predictive information for hepatopathy after the Fontan operation. For example, a high LS value early after completion of a
Fontan procedure might indicate severe hepatic congestion resulting from inappropriate Fontan circulation, even though hepatic fibrosis is not yet present, and would suggest a high risk of congestive hepatopathy developing in a given patient. Additionally, changes in the LS value in patients with chronic stable Fontan circulation might inform us regarding the progress of their liver fibrosis. This postulation did not undergo histologic examinations; therefore, it would seem difficult to conclude which was responsible for the increase in the LS values-aggravation of hepatic congestion or progression of hepatic fibrosis. However, other noninvasive assessments could help to solve this issue. In the present study, the Fontan group had significantly greater LS values than did the RHF group. Also, the results of the coagulation and biochemical tests were worse in the Fontan group compared with those in the RHF group, although this difference was small. From these results, we have concluded that patients with Fontan circulation will have considerable hepatic fibrosis compared with the patients with RHF. 


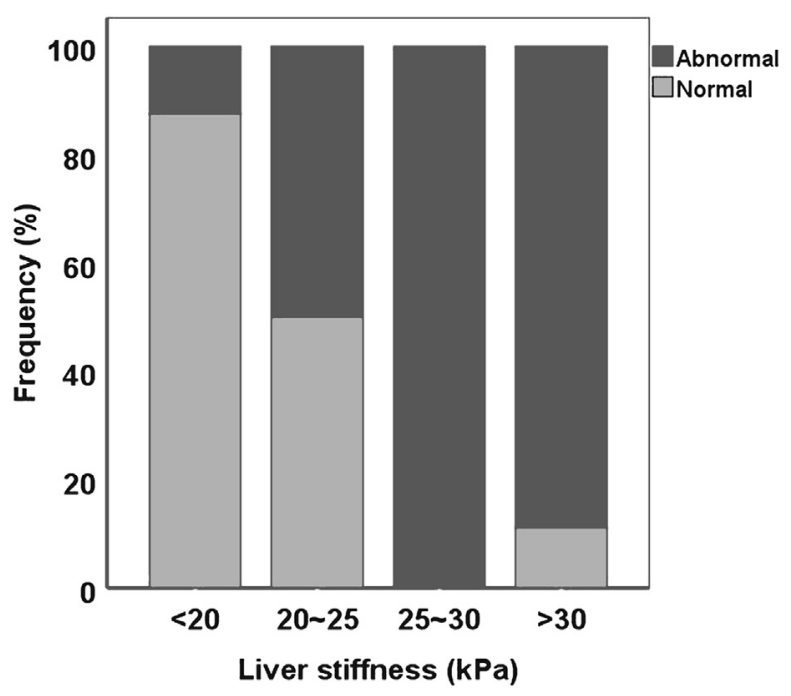

FIGURE 3. Frequency of abnormal ultrasound findings in the Fontan group.

We had intended to investigate the feasibility of TE for assessing hepatopathy in patients with Fontan circulation, even though the LS value will be influenced by the presence of hepatic congestion. Comparing the LS of the Fontan patients with that of a normal population or with another cause of liver disease, we could not interpret whether the LS value of the Fontan group was caused by hepatic fibrosis or congestion. Therefore, we chose a patient group with cardiac-origin chronic liver congestion and investigated the difference in the LS values between the 2 groups. In our results, the LS value was much greater in the Fontan group and correlated with the laboratory test and ultrasound findings for hepatic fibrosis. Thus, we postulated that the greater LS in the Fontan group reflected increased hepatic fibrosis, rather than just congestion and that TE can estimate the severity of hepatic fibrosis in these patients. This postulation requires confirmation from follow-up studies using histologic assessment.

When interpreting a given LS value, it should be considered that the cutoff values used to diagnose advanced fibrosis will differ according to the particular etiology. ${ }^{26}$ A previous study using TE in patients with Fontan circulation categorized the data for the LS value according to the range defined for chronic hepatitis. ${ }^{17}$ The range for TE from biopsy matching studies in chronic hepatitis was 2.5 to 7.5 for absent or mild fibrosis, 7.5 to 9.5 for significant fibrosis, 9.5 to 13.0 for severe fibrosis, and $>13.0$ for cirrhosis. ${ }^{27}$ However, our study showed a very different range of LS values for Fontan patients than those for patients with chronic hepatitis. Most of the patients with Fontan circulation had LS values $>10 \mathrm{kPa}$, and the incidence of liver cirrhosis was significantly increased in the subgroup with LS values $>30 \mathrm{kPa}$. All these findings highlight the need for additional research to determine the new biopsy-based LS cutoff values optimized for patients with Fontan circulation. Previous studies have tried to identify the correlations between congestive hepatopathy after Fontan operation and clinical factors. Although most of these studies were small series, the systemic venous pressure and the duration of Fontan circulation were associated factors in a few studies. ${ }^{6,7,17}$ Also, a low cardiac index and reduced heart rate were suggested as influencing factors, with the hypothesis that reduced hepatic blood flow might induce hypoxic stress and chronic injury in the liver of Fontan patients. ${ }^{23,28}$ In our study, the duration of Fontan circulation and LS values did not correlate significantly but patient age at the completion of the Fontan procedure was an independent factor associated with the LS value on multivariate analysis. Additionally, in Figure 2, $B$, the patients with late Fontan completion (age $>6$ years) showed exaggerated LS compared with the others. Although a previous study using TE in patients with Fontan circulation found a correlation between patient age at Fontan completion and a serum fibrosis marker (FibroTest, licensed by Assistance Publique-Hôptaux de Paris to BioPredictive, Paris, France),${ }^{17}$ many previous studies did not consider age at completion of the Fontan procedure in their analysis. ${ }^{6,22,29}$ Thus, this clinical factor has not been sufficiently discussed. However, it was evident in several previous studies that the hepatopathy associated with Fontan circulation can begin before completion of the Fontan procedure owing to hypoxemia. ${ }^{30,31}$ Although the evidence for preoperative hypoxemia was insufficient in our study, these studies support our suggestion that a late Fontan operation will prolong the hypoxic period, resulting in a hepatic insult and, thus, plays an adverse role in the progression of congestive hepatopathy after the Fontan operation. ${ }^{4,23}$ Because more than one half of the patients in our Fontan group had severe pulmonary outflow obstructive disease (eg, pulmonary atresia or tricuspid atresia) and a systemic to pulmonary shunt history before the Fontan operation, our findings might have revealed the effect of preoperative hypoxia on the liver. However, our postulation requires more supporting evidence and demonstrations of the influence of late Fontan completion on hepatopathy in future prospective studies. Of the patients with a cirrhotic liver on their ultrasound examination, all had had a long Fontan duration (14-29 years) and other unfavorable factors such as older age at Fontan completion (patient 1, 18 years; patient 4, 16 years), low arterial oxygen saturation ( $<75 \%$; patients 1 and 2$)$, and/or serious arrhythmia (patient 1, complete atrioventricular block). However, because of the small number of patients $(n=4)$, we could not find any predictive model for the liver cirrhosis. Although the correlation between the duration of Fontan circulation and the LS value was not statistically significant in our study, that could also have been limited 
by the small number of subjects. Thus, its relationship can only be more clearly revealed in additional studies with a larger number of subjects.

However, the IVC diameter correlated with the LS values in the Fontan group. A previous study revealed that the IVC diameter was associated with hepatic dysfunction, which was assessed by galactose clearance. ${ }^{32}$ This result suggests that IVC dilatation could be a possible marker for congestive hepatopathy after the Fontan operation. However, the correlation between the IVC diameter and LS was not strong in our findings; thus, the presence of congestive hepatopathy cannot be determined by the presence of IVC dilatation. As another marker of the hepatopathy, the splenic size showed a close correlation with the LS value. This result was consistent with a recent study showing that the TE findings and spleen size correlated and could be used to identify portal hypertension. ${ }^{33}$ It is additional evidence of the reliability of TE in assessing Fontan patients.

Although no pathology-based evidence was available to validate the usefulness of TE in detecting hepatopathy in Fontan patients, we believe our results have shown that TE is an easy and useful, noninvasive method for the early detection of hepatopathy and monitoring the disease progression. TE can provide clues to determine the timing for more thorough or invasive examinations and intervention for the liver. Moreover, TE can be used for the assessment of the effect of any potential treatment of hepatopathy.

\section{Study Limitations}

Our study had a relatively small number of subjects, limiting its statistical power. In addition, we assessed congestive hepatopathy using TE and other noninvasive methods, which are not definitive diagnostic tools without confirmation from liver biopsy. Therefore, our study had limitations in validating the reliability of TE and identifying the severity of congestive hepatopathy.

\section{CONCLUSIONS}

The results from the present study have revealed that congestive hepatopathy had developed in a significant fraction of patients with long-term Fontan circulation and showed that TE can be an easy and useful method to assess congestive hepatopathy in Fontan patients. Our study also found that patient age at completion of the Fontan operation is a risk factor for hepatopathy in those with Fontan circulation and that the IVC diameter and spleen size correlated with the presence of congestive hepatopathy. More longitudinal long-term follow-up studies using histologic assessment are warranted to validate the clinical implications for using TE and to determine the specific LS range in patients with Fontan circulation.
We thank C. W. Han and S. J. Song for data gathering and graphic work.

\section{References}

1. Blaufox AD, Sleeper LA, Bradley DJ, Breitbart RE, Hordof A, Kanter RJ, et al. Functional status, heart rate, and rhythm abnormalities in 521 Fontan patients 6 to18 years of age. J Thorac Cardiovasc Surg. 2008;136:100-7.

2. Nakamura Y, Yagihara T, Kagisaki K, Hagino I, Kobayashi J. Ventricular performance in long-term survivors after Fontan operation. Ann Thorac Surg. 2011;91: 172-80.

3. Pike NA, Evangelista LS, Doering LV, Koniak-Griffin D, Lewis AB, Child JS. Clinical profile of the adolescent/adult Fontan survivor. Congenit Heart Dis. 2011;6:9-17.

4. Wu FM, Ukomadu C, Odze RD, Valente AM, Mayer JE Jr, Earing MG. Liver disease in the patient with Fontan circulation. Congenit Heart Dis. 2011;6:190-201.

5. Lemmer JH, Coran AG, Behrendt DM, Heidelberger KP, Stern AM. Liver fibrosis (cardiac cirrhosis) five years after modified Fontan operation for tricuspid atresia. J Thorac Cardiovasc Surg. 1983;86:757-60.

6. Ghaferi AA, Hutchins GM. Progression of liver pathology in patients undergoing the Fontan procedure: chronic passive congestion, cardiac cirrhosis, hepatic adenoma, and hepatocellular carcinoma. J Thorac Cardiovasc Surg. 2005;129: 1348-52.

7. Kiesewetter CH, Sheron N, Vettukattill JJ, Hacking N, Stedman B, MillwardSadler H, et al. Hepatic changes in the failing Fontan circulation. Heart. 2007; 93:579-84.

8. Kendall TJ, Stedman B, Hacking N, Haw M, Vettukattill JJ, Salmon AP, et al. Hepatic fibrosis and cirrhosis in the Fontan circulation: a detailed morphological study. J Clin Pathol. 2008;61:504-8.

9. Bravo AA, Sheth SG, Chopra S. Liver biopsy. N Engl J Med. 2001;344:495-500.

10. Cadranel JF, Rufat P, Degos F, for the Group of Epidemiology of the French Association for the Study of the Liver (AFEF). Practices of liver biopsy in France: results of a prospective nationwide survey. Hepatology. 2000;32:477-81.

11. Regev A, Berho M, Jeffers LJ, Milikowski C, Molina EG, Pyrsopoulos NT, et al. Sampling error and intraobserver variation in liver biopsy in patients with chronic HCV infection. Am J Gastroenterol. 2002;97:2614-8.

12. Sandrin L, Fourquet B, Hasquenoph JM, Yon S, Fournier C, Mal F, et al. Transient elastography: a new noninvasive method for assessment of hepatic fibrosis. Ultrasound Med Biol. 2003;29:1705-13.

13. Nguyen-Khac E, Capron D. Noninvasive diagnosis of liver fibrosis by ultrasonic transient elastography (FibroScan). Eur J Gastroenterol Hepatol. 2006;18:1321-5.

14. Berzigotti A, Ashkenazi E, Reverter E, Abraldes JG, Bosch J. Non-invasive diagnostic and prognostic evaluation of liver cirrhosis and portal hypertension. Dis Markers. 2011;31:129-38.

15. Jung KS, Kim SU, Ahn SH, Park YN, Kim do Y, Park JY, et al. Risk assessment of hepatitis B virus-related hepatocellular carcinoma development using liver stiffness measurement (FibroScan). Hepatology. 2011;53:885-94.

16. European Association for the Study of the Liver. EASL Clinical Practice Guidelines: management of hepatitis C virus infection. J Hepatol. 2011;55:245-64.

17. Friedrich-Rust M, Koch C, Rentzsch A, Sarrazin C, Schwarz P, Herrmann E, et al. Noninvasive assessment of liver fibrosis in patients with Fontan circulation using transient elastography and biochemical fibrosis markers. J Thorac Cardiovasc Surg. 2008;135:560-7.

18. Rudski LG, Lai WW, Afilalo J, Hua L, Handschumacher MD, Chandrasekaran K, et al. Guidelines for the echocardiographic assessment of the right heart in adults: a report from the American Society of Echocardiography endorsed by the European Association of Echocardiography, a registered branch of the European Society of Cardiology, and the Canadian Society of Echocardiography. J Am Soc Echocardiogr. 2010;23:685-713.

19. Kim SU, Lee JH, Kim Do Y, Ahn SH, Jung KS, Choi EH, et al. Prediction of liverrelated events using FibroScan in chronic hepatitis B patients showing advanced liver fibrosis. PLoS One. 2012; 7:e36676.

20. Lin ZH, Xin YN, Dong QJ, Wang Q, Jiang XJ, Zhan SH, et al. Performance of the aspartate aminotransferase-to-platelet ratio index for the staging of hepatitis $\mathrm{C}$ related fibrosis: an updated meta-analysis. Hepatology. 2011;53:726-36.

21. Wai CT, Greenson JK, Fontana RJ, Kalbfleisch JD, Marrero JA, Conjeevaram HS, et al. A simple noninvasive index can predict both significant fibrosis and cirrhosis in patients with chronic hepatitis C. Hepatology. 2003;38:518-26.

22. Baek JS, Bae EJ, Ko JS, Kim GB, Kwon BS, Lee SY, et al. Late hepatic complications after Fontan operation: non-invasive markers of hepatic fibrosis and risk factors. Heart. 2010;96:1750-5. 
23. Roulot D, Czernichow S, Le Clesiau H, Costes JL, Vergnaud AC, Beaugrand M. Liver stiffness values in apparently healthy subjects: influence of gender and metabolic syndrome. J Hepatol. 2008;48:606-13.

24. Millonig G, Friedrich S, Adolf S, Fonouni H, Golriz M, Mehrabi A, et al. Liver stiffness is directly influenced by central venous pressure. J Hepatol. 2010;52: 206-10.

25. Hopper I, Kemp W, Porapakkham P, Sata Y, Condon E, Skiba M, et al. Impact of heart failure and changes to volume status on liver stiffness: non-invasive assessment using transient elastography. Eur J Heart Fail. 2012;14:621-7.

26. Wong VW, Chan HL. Transient elastography. J Gastroenterol Hepatol. 2010;25: 1726-31.

27. Castera L. Invasive and non-invasive methods for the assessment of fibrosis and disease progression in chronic liver disease. Best Pract Res Clin Gastroenterol. 2011;25:291-303.

28. Camposilvan S, Milanesi O, Stellin G, Pettenazzo A, Zancan L, D’Antiga L. Liver and cardiac function in the long term after Fontan operation. Ann Thorac Surg. 2008;86:177-82.
29. Ginde S, Hohenwalter MD, Foley WD, Sowinski J, Bartz PJ, Venkatapuram S et al. Noninvasive assessment of liver fibrosis in adult patients following the Fontan procedure. Congenit Heart Dis. 2012;7:235-42.

30. Odegard KC, McGowan FX Jr, DiNardo JA, Castro RA, Zurakowski D, Connor CM, et al. Coagulation abnormalities in patients with single-ventricle physiology precede the Fontan procedure. J Thorac Cardiovasc Surg. 2002; 123:459-65

31. Schwartz MC, Sullivan L, Cohen MS, Russo P, John AS, Guo R, et al. Hepatic pathology may develop before the Fontan operation in children with functional single ventricle: an autopsy study. J Thorac Cardiovasc Surg. 2012; 143:904-9.

32. Narkewicz MR, Sondheimer HM, Ziegler JW, Otanni Y, Lorts A, Shaffer EM, et al. Hepatic dysfunction following the Fontan procedure. J Pediatr Gastroenterol Nutr. 2003;36:352-7.

33. Berzigotti A, Seijo S, Arena U, Abraldes JG, Vizzutti F, García-Pagán JC, et al Elastography, spleen size, and platelet count identify portal hypertension in patients with compensated cirrhosis. Gastroenterology. 2013;144:102-11. 